\title{
Motor Accessibility of Smartwatch Touch and Bezel Input
}

\author{
Meethu Malu' \\ ${ }^{1}$ Computer Science, ${ }^{2}$ Information Studies \\ University of Maryland, College Park, MD, USA \\ meethu@cs.umd.edu,pchundur@umd.edu
}

Pramod Chundury ${ }^{2}$

Leah Findlater ${ }^{3}$

\author{
${ }^{3}$ Human Centered Design \& Engineering \\ University of Washington, Seattle, WA, USA \\ leahkf@uw.edu
}

\begin{abstract}
Smartwatches present inherent input difficulties due to the small touchscreen. In a controlled experiment with 14 participants with upper body motor impairments, we compared smartwatch touchscreen input to input on the bezel of the watch, the latter of which should at least theoretically stabilize user input due to its hard edge. Results demonstrate a speed-accuracy tradeoff whereby the touchscreen is faster but the bezel is more accurate.
\end{abstract}

\section{Author Keywords}

Smartwatch; motor impairment; touchscreen; accessibility.

\section{ACM Classification Keywords}

- Human-centered computing Accessibility systems and tools.

\section{INTRODUCTION AND BACKGROUND}

Smartwatches present an inherent accessibility challenge: the small screen, often only $\sim 4 \mathrm{~cm}$ in width, requires precise input and can be difficult for people with upper body motor impairments to use [6]. Touchscreen accessibility work, however, has largely focused on smartphones and tablets, showing, for example, that people with motor impairments encounter higher error rates [2, 8] and exhibit longer dwell times than people without motor impairments [4]. Multi-touch gestures and text entry can also be particularly difficult $[1,5,10]$.

One strategy to address these challenges is to stabilize the user's finger by utilizing the hard edges of the screen [3, 12]. While modern smartwatches do not have the same hard screen edges as older mobile devices, taps or swipes on the bezel (Figure 1) rather than the touchscreen may provide similar benefits while also mitigating the fat-finger problem (a common issue with small screens [9]). Indeed, a study by Malu et al. [6] showed that users with motor impairments were open to the idea of bezel gestures and preferred them to other non-touchscreen input options (skin or wristband input). However, that qualitative study did not measure users' input performance with the bezel.

We report on a controlled lab study comparing touchscreen and bezel input with 14 participants with upper body motor

Permission to make digital or hard copies of part or all of this work for personal or classroom use is granted without fee provided that copies are not made or distributed for profit or commercial advantage and that copies bear this notice and the full citation on the first page. Copyrights for thirdparty components of this work must be honored. For all other uses, contact the Owner/Author.

ASSETS '19, October 28-30, 2019, Pittsburgh, PA, USA

(C) 2019 Copyright is held by the owner/author(s).

ACM ISBN 978-1-4503-6676-2/19/10.

https://doi.org/10.1145/3308561.3354638
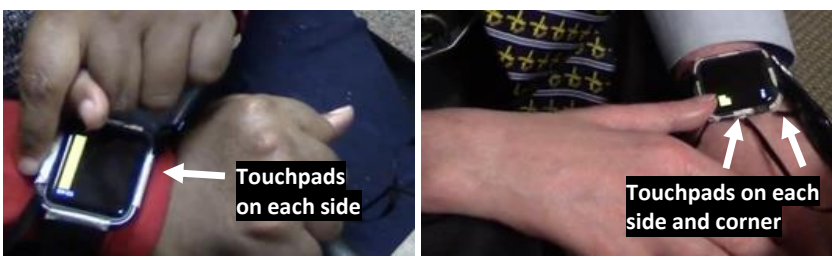

Figure 1. Bezel input: four (left) or eight (right) conductive fabric touchpads were affixed to a smartwatch case.

impairments. Our findings reveal a speed-accuracy tradeoff: the bezel significantly lowered error rates with small targets, but the touchscreen was significantly faster. We discuss the implications of these findings and subjective feedback, and outline next steps to build on this research.

\section{METHOD}

Our controlled experiment compared bezel and touchscreen input performance for two target layouts (4 and 8 targets).

\section{Participants}

Fourteen participants (6 women, 8 men) with upper body motor impairments were recruited. They were on average 36.9 years old $(S D=13.6)$ and their most common diagnosed medical conditions were cerebral palsy $(N=5)$, muscular dystrophy $(N=2)$, and spinal muscular atrophy $(N=2)$. On a standardized Box-and-Block test of gross manual dexterity, scores ranged from 0-48 (adults without motor impairments score $~ 80$ [7]). All owned a smartphone, two owned a smartwatch, and one owned a wrist-worn fitness tracker.

\section{Apparatus and Procedure}

We built a testbed in Swift for a $42 \mathrm{~mm}$ Apple Watch 1 (Figure 2). While the screen was $24 \times 27 \mathrm{~mm}$, we restricted the active area to $24 \times 24 \mathrm{~mm}$ so the vertical and horizontal spans were equal. For touchscreen input, this area was divided into a $2 \times 2$ grid for the 4-target layout and a 4x2 grid for the 8-target condition (mimicking common layouts in watchOS). For bezel input, we affixed conductive fabric to a smartwatch case (Figure 1). These touchpads were wired to an Arduino Uno that connected via Bluetooth to an iPhone $5 \mathrm{~s}$ paired to the watch. For the 4-target condition, we centered a $24 \mathrm{~mm}$-long target along each side of the case (this length was chosen to match the active touchscreen size rather than the full watch bezel); touchpads were $5 \mathrm{~mm}$ in height. For the 8-target layout, eight $12 \mathrm{~mm}$-long touchpads were centered on the sides and corners of the case.

Study sessions were 90 minutes and began with background questions and the Box-and-Block test. Then, the four experimental conditions were presented in counterbalanced order using a balanced Latin Square. For each condition, 

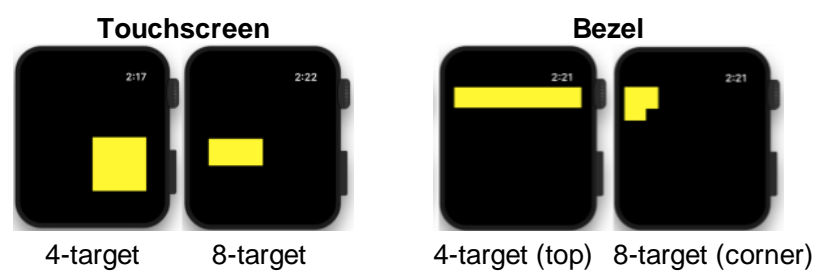

Figure 2. Visual cues. Participants tapped directly on the touchscreen target (left) or on the closest bezel target (right).

participants completed a practice block (8 tapping trials) followed by three test blocks (16 trials each) with brief rests between blocks. Within each block, each target location was presented the same number of times (e.g., 2 times for the 8-target layout and 4 times for the 4-target layout), and trials were randomly ordered such that a single target would not be appear twice in a row. For each trial, a visual cue was shown (Figure 2) and an audio cue played. A trial ended upon successfully tapping the target or after a 10second timeout (indicating substantial difficulty). The session concluded with semi-structured questions.

\section{Study Design, Data and Analysis}

We used a $2 \times 2$ within-subjects design: technique (bezel $v s$. touchscreen) $\times$ layout (4- vs. 8-target). We hypothesized that bezel input would be faster $(\mathrm{H} 1)$ and more accurate (H2) than touchscreen input. The dependent variables of time and error rate violated the normality assumption of ANOVAs (shown by Q-Q plots and Shapiro-Wilk's W tests), so we used $2 \times 2$ repeated measures (technique $\times$ layout) ANOVAs with Aligned Rank Transform-a nonparametric alternative [11]. Posthoc pairwise comparisons used Bonferroni-adjusted Wilcoxon signed rank tests.

\section{RESULTS}

Overall, we found a speed-accuracy tradeoff, as shown in Figure 3. The touchscreen was significantly faster $(M=$ $1.2 \mathrm{~s}, S D=0.2)$ than the bezel $(M=1.7 \mathrm{~s}, S D=0.2)$ (main effect of technique: $F_{1,13}=22.60, p<.001, \eta_{p}^{2}=.63$ ). Reflecting differences in target size, the 4-target layout was significantly faster than the 8-target layout, at on average $1.3 \mathrm{~s}(S D=0.4)$ versus $1.6 \mathrm{~s}(S D=0.4)$ (main effect of layout: $\left.F_{1,13}=50.50, p<.001, \eta_{p}^{2}=.79\right)$. The layout $\mathrm{x}$ technique interaction effect was not significant for time.

In contrast, the bezel yielded a significantly lower error rate $(M=3.5 \%, S D=1.0)$ than the touchscreen $(M=10.0 \%, S D$ $=11.3$ ) (main effect of technique: $F_{1,13}=18.62, p<.001$, $\left.\eta_{p}^{2}=.58\right)$. As expected again, the 4-target layout resulted in a significantly lower error rate $(M=2.5 \%, S D=0.5)$ than the 8-target layout $(M=11.1 \%, S D=9.79)$ (main effect of layout: $\left.F_{1,13}=26.54, p<.001, \eta_{p}^{2}=.67\right)$. However, there was also a significant technique $\times$ layout interaction effect $\left(F_{1,13}=37.20, p<.001, \eta_{p}^{2}=.74\right)$. Posthoc pairwise comparisons showed that the touchscreen was particularly error prone with the small targets in the 8-target layout compared to the bezel $(p<.05)$, but that the two types of input were not different for the 4-target layout $(p>.05)$.
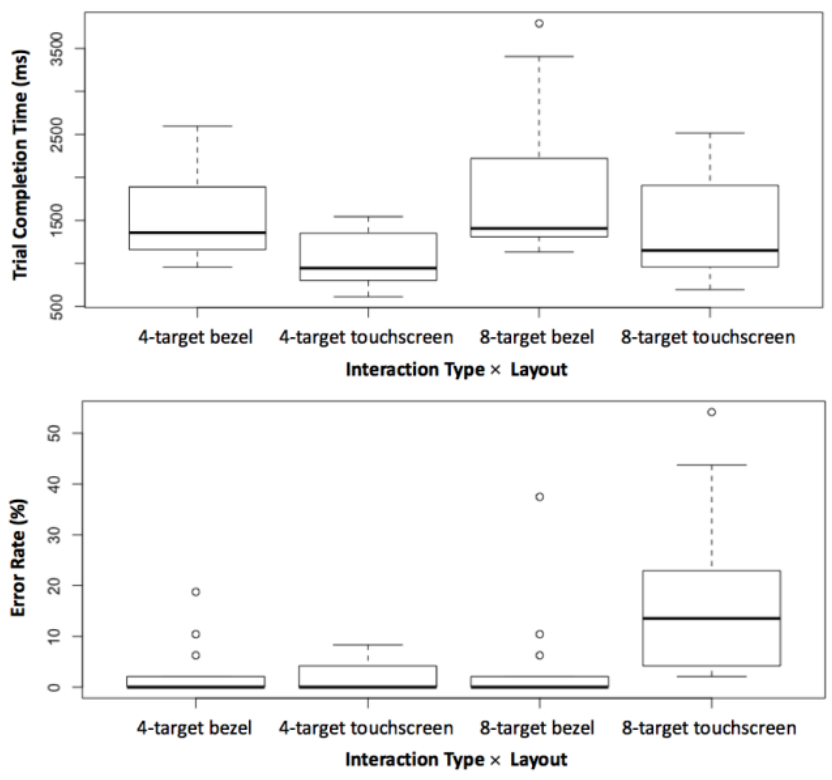

Figure 3. Boxplots of average trial completion time (top) and error rate (bottom). Lower values are better in both graphs.

Subjectively, participants largely favored the touchscreen despite its higher error rate. For example, all but one participant felt the touchscreen was more comfortable (e.g., due to familiarity, did not require twisting the wrist) and easier to use (e.g., due to the flat surface and combined input/output space). However, participants also identified advantages to bezel input, such as the ability to use multiple fingers ("I found myself using only the index finger for the touchscreen but for the bezel I was using multiple fingers", P8), and different finger orientations ( "different sides of my finger", P5). Four participants also felt the bezel could be useful for quick tasks (e.g., stopwatch start/stop, P9), three thought it could be useful when occlusion is a problem (e.g., manipulating a map), and one participant (P6) suggested the bezel could be a more accessible alternative to the physical buttons on the side of the watch.

\section{DISCUSSION AND FUTURE WORK}

Our findings reveal a speed-accuracy tradeoff that supports hypothesis $\mathrm{H} 2$ but not $\mathrm{H} 1$ : the touchscreen is faster but the bezel is more accurate, particularly for small targets. While participants largely preferred the touchscreen for general input, the bezel could be useful for specific scenarios, such as when needing to limit visual occlusion, performing quick shortcut gestures, or as an alternative to small physical buttons. Several possibilities exist to improve the bezel input, such as increasing the size of the touchpads, adding physical guides (e.g., notches) to stabilize input, only using the watch sides that are within easiest reach, and exploring swiping as well as tapping. Ultimately, while the bezel had previously shown promise for accessible off-screen input [6], we recommend using it as a complement to the touchscreen and focusing on further design tweaks.

\section{ACKNOWLEDGMENTS}

This research was funded by NSF grant IIS-1350438. 


\section{REFERENCES}

1. Lisa Anthony, YooJin Kim, and Leah Findlater. 2013. Analyzing user-generated youtube videos to understand touchscreen use by people with motor impairments. Proc. CHI '13, 1223-1232. http://doi.org/10.1145/2470654.2466158

2. S. N. Duff, C. B. Irwin, J. L. Skye, M. E. Sesto, et al. 2010. The Effect of Disability and Approach on Touch Screen Performance during a Number Entry Task. Proceedings of the Human Factors and Ergonomics Society Annual Meeting 54, 6: 566-570. http://doi.org/10.1177/154193121005400605

3. Jon Froehlich, Jacob O. Wobbrock, and Shaun K. Kane. 2007. Barrier pointing: using physical edges to assist target acquisition on mobile device touch screens. Proc. ASSETS '07, 19-26. http://doi.org/10.1145/1296843.1296849

4. Curt B. Irwin and Mary E. Sesto. 2012. Performance and touch characteristics of disabled and non-disabled participants during a reciprocal tapping task using touch screen technology. Applied Ergonomics 43, 6: 1038-1043. http://doi.org/10.1016/j.apergo.2012.03.003

5. Shaun K. Kane, Chandrika Jayant, Jacob O. Wobbrock, and Richard E. Ladner. 2009. Freedom to roam: a study of mobile device adoption and accessibility for people with visual and motor disabilities. Proc. ASSETS '09, 115-122. http://doi.org/10.1145/1639642.1639663

6. Malu, Meethu, Chundury, Pramod, and Findlater, Leah (2018, April). Exploring accessible smartwatch interactions for people with upper body motor impairments. Proceedings of the 2018 CHI Conference on Human Factors in Computing Systems (paper 488). http://doi.org/10.1145/3173574.3174062
7. V Mathiowetz, G Volland, N Kashman, and K Weber. 1985. Adult norms for the Box and Block Test of manual dexterity. The American journal of occupational therapy : official publication of the American Occupational Therapy Association 39, 6: 386-91. Retrieved March 7, 2017 from http://www.ncbi.nlm.nih.gov/pubmed/3160243

8. Kyle Montague, Hugo Nicolau, and Vicki L. Hanson. 2014. Motor-impaired touchscreen interactions in the wild. Proc. ASSETS '14, 123-130. http://doi.org/10.1145/2661334.2661362

9. Shaikh Shawon Arefin Shimon, Courtney Lutton, Zichun Xu, Sarah Morrison-Smith, Christina Boucher, and Jaime Ruiz. 2016. Exploring Non-touchscreen Gestures for Smartwatches. Proceedings of the 2016 CHI Conference on Human Factors in Computing Systems - CHI '16, ACM Press, 3822-3833. http://doi.org/10.1145/2858036.2858385

10. Shari Trewin, Cal Swart, and Donna Pettick. 2013. Physical accessibility of touchscreen smartphones. Proc. ASSETS '13, 1-8. http://dx.doi.org/10.1145/2513383.2513446

11. Jacob O. Wobbrock, Leah Findlater, Darren Gergle, and James J. Higgins. 2011. The aligned rank transform for nonparametric factorial analyses using only anova procedures. Proceedings of the 2011 annual conference on Human factors in computing systems - CHI '11, ACM Press, 143. http://doi.org/10.1145/1978942.1978963

12. Jacob O. Wobbrock, Brad A. Myers, and John A. Kembel. 2003. EdgeWrite: a stylus-based text entry method designed for high accuracy and stability of motion. Proc. UIST '03, 61-70. http://doi.org/10.1145/964696.964703 